\title{
The Proof of The Beal's Conjecture
}

\author{
Leszek W. Guła \\ Lublin-POLAND
}

\begin{abstract}
Keywords Common Prime Factor, Diophantine Equations, Fermat Equation, Greatest Common Divisor, Newton Binomial Formula.
\end{abstract}

\section{Abstract}

1. The truly marvellous proof of The Fermat's Last Theorem (FLT).

2. Two false proofs of FLT for $n=4$.

3. The incomplete proof of FLT for odd prime numbers $n \in \mathbb{P}$.

4. The proof of The Beal's Conjecture.

5. The Beal's Theorem.

\section{Dedicatory}

Dedicated to my Parents and my Brother

\section{INTRODUCTION}

The cover of this issue of the Bulletin is the fron spiece to a volume of Samuel de Fermat's 1670 edition of Bachet's Latin translation of Diop ntus's Ari hmetica. This edition includes the marginalia of the editor's father, Pierre de Ferma 'mong nese notes one finds the elder Fermat's extraordinary comment in connectio the Pyuragorean equation $x^{2}+y^{2}=z^{2}$ the marginal comment that hints at the existence of pro domonstratio sane mirabilis) of what has come to be known as Fermat's Last Theorem. Diophay us's work had fired the imagination of the Italian Renaissance mathematican ael Bon belli, as it inspired Fermat a century later. [6]

Problem II.8 of the Diophan Ar ametica sks how a given square number is split into two other squares. Diophantus's s ons hu to sorvethis sum-of-squares problem for $k=4$ and $u=2$ [8], inasmuch as for all

$$
\left.\left.k^{2}=\left(\frac{2 k u}{u^{2}+1}\right)^{2} \cdot \frac{k(u-}{u^{2}+1}\right)\right]^{2},[4]
$$

Thus for all relativ prime $n$ cural numbers $u, v$ such that $u-v \in\{1,3,5, \ldots\}$ : $\left(u^{2}+y^{2}\right)^{4}-2 u v^{4}+4 u^{2} v^{2}=\left(u^{2}-v^{2}\right)^{2}+(2 u v)^{2}$.

We ná because th mbers $x, y$, and $z$ are coprime.

Around 1637, Crmat wrote his Last Theorem in the margin of his copy of the Arithmetica next to Diophantus sum-of-squares problem: it is impossible to separate a cube into two cubes, or a fourth power into two fourth powers, or in general, any power higher than the second, into two like powers. I have discovered a truly marvelous proof of this, which this margin is too narrow to contain. In number theory, Fermat's Last Theorem (FLT) states that no three positive integers $A, B$, and $C$ can satisfy the equation $A^{n}+B^{n}=C^{n}$ for any integer value of $n$ greater than two. [8] It is easy to see that if $A^{n}+B^{n}=C^{n}$ then either $A, B$, and $C$ are co-prime or, if not co-prime that any common factor could be divided out of each term until the equation existed with co-prime bases. (Co-prime is synonymous with pairwise relatively prime and means that in a given set of numbers, no two of the numbers share a common factor). 
You could then restate FLT by saying that $A^{n}+B^{n}=C^{n}$ is impossible with co-prime bases. (Yes, it is also impossible without co-prime bases, but non co-prime bases can only exist as a consequence of co-prime bases). [1]

It is known that for some coprime $x, y, z \in\{3,4,5, \ldots\}$ :

$\left[x^{2}+y^{2}=z^{2} \wedge(x+y)^{2}+(x-y)^{2}=2 z^{2}\right]$,

where $Z$ is odd because for all $a, b \in\{0,1,2,3, \ldots\}$ : the number $\frac{(2 a+1)^{2}+(2 b+1)^{2}}{2}$ is odd.

\section{THE FERMAT'S LAST THEOREM AND THE BEAL'S CONJECTURE}

Theorem 1 (FLT). For all $n \in\{3,4,5, \ldots\}$ and for all $A, B, C \in\{1,2,3, \ldots\}: B^{n} \neq{ }^{2}$.

Conjecture 1. For some $x, y, z \in\{3,4,5 \ldots\}$ and for some $A, B, C \in\{1,2, \ldots\}$ for ome prime number (a common prime factor) $\mathbf{p} \geq 2$ :

$\left(A^{x}+B^{y}=C^{z} \wedge \mathbf{p} \mid A, B, C\right)$.

Or - For all $x, y, z \in\{3,4,5, \ldots\}$ and for all coprime $A, B, C \in\{3, \ldots\}$ :

$\left(A^{x}+B^{y} \neq C^{z}\right)$.

This is The Beal's Conjecture (slightly restated).

\section{THE TRULY MARVELLOUS PROOF OF FLT}

Proof. Every even number which is not the of numver 2 has odd prime divisor, hence sufficient that we prove FLT for $\mathrm{n}=4$ and for $\mathrm{o} \mathrm{d} \mathrm{p}$ imbers $n \in \mathbb{P}$.

Suppose that for $(n=4 \vee n \in \mathbb{P})$ for som coprime $A, B, C \in\{1,2,3, \ldots\}$ :

$\left(A^{n}+B^{n}=C^{n} \wedge A+B>C \wedge 2+B>C^{2} \wedge \cdots \wedge A^{n-1}+B^{n-1}>C^{n-1}\right)$,

otherwise $A^{n}+B^{n}<C^{n}$.

Then only one nuy wer out of ( $g$ is even and the even number $A+B-C>0$ and for $n=4$ the num $C$ is dd in view of (1).

Without loss for th. roof we Can assume that $A, C-B \in\{1,3,5, \ldots\}$ and $4 \nmid B, C$.

A. The

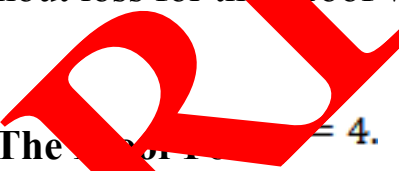

For some copine $B, C, A \in\{1,2,3, \ldots\}$ such that $C, C-B, A \in\{1,3,5, \ldots\}$ and $B<C>A$ :

$\left[B-(C-A)=2 v \wedge(C-A+2 v)^{4}=B^{4}=(C-A+A)^{4}-A^{4}\right] \Rightarrow$

$(C-A)^{2} 2 v+6(C-A) v^{2}+8 v^{3}+\frac{4 v^{4}}{C-A}=(C-A)^{2} A+\frac{3}{2}(C-A) A^{2}+A^{3}$.

Thus for some $h, v, B, C, A \in\{1,3,5, \ldots\}$ :

$\left(4 h^{4}+2 v=B \wedge 4 h^{4}=C-A \wedge h \mid v\right)$. 
By analogy we get - for some $c, h, A, C \in\{1,3,5, \ldots\}$ :

$\left(c^{4}+2 c h=A \wedge c h=v \wedge c^{4}=C-B\right)$.

Hence for some relatively prime $c, h \in\{1,3,5, \ldots\}$ :

$\left(2 c h+4 h^{4}\right)^{4}=\left[\left(4 h^{4}+A\right)^{2}\right]^{2}-\left(A^{2}\right)^{2}=\left[\left(4 h^{4}+A\right)^{2}+A^{2}\right]\left(2 h^{4}+A\right) 8 h^{4}$,

whence it implies that for some $z, w, x \in\{1,3,5, \ldots\}$ and for some $y \in\{6,10,14, \ldots\}$ :

$\left[z w=c+2 h^{3} \wedge x+y=2 h^{4}+A+2 h^{4} \wedge x=2 h^{4}+A \wedge y=2 h^{4} \wedge\right.$

$4 \nmid y \wedge 2(z w)^{4}=\left((x+y)^{2}+(x-y)^{2}\right) x=2\left(x^{2}+y^{2}\right) x \wedge$ $\left.z^{4} w^{4}=\left(x^{2}+y^{2}\right) x \wedge\left(z^{2}\right)^{2}=\left(x^{2}+y^{2}\right) \wedge w^{4}=x\right] \Rightarrow 4 \mid y$, which is inconsistent with $4 \nmid y$. This is the proof for $n=4$.

B. The Proof For $n \in \mathbb{P}$. General Deductions.

For some $n \in \mathbb{P}$ and some coprime $A, C, B \in\{1,2,3, \ldots\}$ suc that $-B \in \lambda(1,3,5, \ldots\}$ :

$[A-(C-B)=B-(C-A)=2 v \wedge C-B+2 v=A \wedge C-A+2 v=/ \wedge$

$(C-B+2 v)^{n}=(C-B+B)^{n}-B^{n} \wedge\left(C-A+2 v=(C-A-A)^{n}-A^{n} \wedge\right.$

$\left.(A+B-B)^{n}+B^{n}=(A+B-2 v)^{n}=C^{n}\right]$

Hence for some coprime $C, B, A \in\{1,2,3, \ldots\}$ S $C-B, A \in\{1,3,5, \ldots\}$ :

$$
\begin{aligned}
& \left\{(C-B)^{n-2} v+(n-1)\left(C-v^{2}+2^{n-2} v^{n-1}+\frac{2^{n-1} v^{n}}{n(C-B)}=\right.\right. \\
& \frac{B}{2}\left[(C-B)^{n-2}+\frac{n-1}{2}\right) B \\
& (C-A)^{n-2} 2 v+\frac{n}{2}(C-4)^{n-3}(2 v)^{2}+\cdots+(2 v)^{n-1}+\frac{(2 v)^{n}}{n(C-A)}= \\
& A\left[(C-A)^{2} \frac{n}{2}(-A)^{n-3} A+\cdots+A^{n-2}\right] \wedge \\
& (A+5)^{n-}(-B)+\frac{-1}{2}(A+B)^{n-3}(-B)^{2}+\cdots+(-B)^{n-1}= \\
& \left.\left.(A+B)^{n}=2 v\right)+\frac{n-1}{2}(A+B)^{n-3}(-2 v)^{2}+\cdots+(-2 v)^{n-1}+\frac{(-2 v)^{n}}{n(A+B)}\right\} \text {. }
\end{aligned}
$$

Thus for some $n \in \mathbb{P}$ and for some $m, c, h, A, C-B \in\{1,3,5, \ldots\}$ :

$\left[n m c h=v \wedge n \nmid m c h \wedge n, C-A, A+B, C-B \mid(2 v)^{n} \wedge\right.$

$(n|A, C-B \vee n| B, C-A \vee n \mid A+B, C) \wedge(4 \nmid B \wedge 4 \nmid C) \equiv 4 \nmid B, C]$. 
B.1. The Proof For Odd $A, B, C-B$.

For some $n \in \mathbb{P}$ and some $m, c, h \in\{1,3,5, \ldots\}$ with $n \nmid m c h$ :

$\left[\left(n^{n-1} c^{n}+2 n m c h=A \wedge n \mid A \wedge h^{n}+2 n m c h=B \wedge\right.\right.$

$\left.2^{n} m^{n}=A+B=n^{n-1} c^{n}+h^{n}+4 n m c h \wedge n^{n-1} c^{n}+B=C\right) \vee$

$\left(c^{n}+2 n m c h=A \wedge n \mid B \wedge n^{n-1} h^{n}+2 n m c h=B \wedge\right.$

$\left.2^{n} m^{n}=A+B=c^{n}+n^{n-1} h^{n}+4 n m c h \wedge c^{n}+B=C\right) \vee$

$\left(c^{n}+2 n m c h=A \wedge n \mid A+B, C \wedge h^{n}+2 n m c h=B \wedge\right.$

$2^{n} n^{n-1} m^{n}=A+B=c^{n}+h^{n}+4 n m c h \wedge c^{n}+B=C$

$\left[\left(2^{n} m^{n}-h^{n}=n^{n-1} c^{n}+4 n m c h \wedge n \mid 2 m-h \wedge\right.\right.$

$\left(2^{n} m^{n}-c^{n}=n^{n-1} h^{n}+4 n m c h \wedge n \mid 2 m-c\right.$

$\left.\left(2^{n} n^{n-1} m^{n}=c^{n}+h^{n}+4 n m c h \wedge n \mid c+\left\langle n^{2}\right| c n^{n}\right)\right] \Longrightarrow n \mid m c h$,

which is isconsistent with $n \nmid m c h$.

B.2. The Proof For Even $B, C-A$.

For some $n \in \mathbb{P}$ and some $m, c, h$. $\}$ with $n \nmid m c h$ :

$\left[\left(n^{n-1} c^{n}+2 n m c h=A \wedge \wedge \wedge \wedge h^{n}+2 n m c h=B \wedge\right.\right.$

$\left.m^{n}=A+B=n^{n-1}, n m c h \wedge n^{n-1} c^{n}+B=C\right) \vee$

$\left(c^{n}+2 n m c h=A \wedge n|B\rangle \mathbb{2}^{n} n^{n-1} h^{n}+2 n m c h=B \wedge\right.$

$m^{n}=A+B=\left(2^{n}, y^{-1} h^{n}+4 n m c h \wedge c^{n}+B=C\right) \vee$

$\left(c^{n}+2 n m c \cdot A \wedge n \mu^{A}+B, C \wedge 2^{n} h^{n}+2 n m c h=B \wedge\right.$

$\left.\left.n^{n-1} m^{n}+B \quad c^{n}+2^{n} h^{n}+4 n m c h \wedge c^{n}+B=C\right)\right] \Rightarrow$

$\left[\left(m-2^{n}-n^{n} c^{n}+4 n m c h \wedge n\left|m-2 h \wedge n^{2}\right| m^{n}-2^{n} h^{n}\right) \vee\right.$

$\left(m^{n}-c \wedge 2^{n} n^{n-1} h^{n}+4 n m c h \wedge n\left|m-c \wedge n^{2}\right| m^{n}-c^{n}\right) \vee$

$\left.\left(n^{n-1} m^{n}=c^{n}+2^{n} h^{n}+4 n m c h \wedge n\left|c+2 h \wedge n^{2}\right| c^{n}+2^{n} h^{n}\right)\right] \Rightarrow n \mid m c h$,

which is isconsistent with $n \nmid m c h$. This is the proof. 


\section{TWO FALSE PROOF OF FLT FOR $n=4$ WITH $4 \mid \mathrm{B}$}

False Proof 1. For some $u, v \in\{1,2,3, \ldots\}$ and for some $A, C, a, k, b, c \in\{1,3,5, \ldots\}$ and for some $B \in\{6,10,14, \ldots\}$ such that $u-v \in\{1,3,5, \ldots\}$ and $k>1$ and $C>a>2^{\frac{k}{2}} b c$ and $u, v, A, C$ are coprime and $A, C, a, b, c$ are coprime:

$\left\{\left[u^{2}-v^{2}=A^{2} \wedge u^{2}+v^{2}=C^{2} \wedge u=a^{2} \wedge v=2^{k} b^{2} c^{2} \wedge 2 u v=B^{2} \wedge\right.\right.$

$\left.\left.2 u^{2}=C^{2}+A^{2} \wedge a^{4}+\left(2^{\frac{k}{2}} b c\right)^{4}=C^{2} \equiv 0 \wedge\left(A^{2}\right)^{2}+\left(B^{2}\right)^{2}=\left(C^{2}\right)^{2}\right] \in \mathbf{0}\right\}$,

inasmuch as $2^{\frac{k}{2}} \notin\{1,3,5, \ldots\}$.

Moreover on the strength of (1):

$(C=A+v \wedge \pm A=A-v) \Rightarrow[(v=0 \vee v=2 A) \wedge \operatorname{gcd}(v, A)>$

which is inconsistent with $[v>0 \wedge \operatorname{gcd}(v, A)=1]$. This is the f e proof 1 .

False Proof 2. For some relatively prime $u, v \in\{1,2,3, \ldots\}$ an fo, me $C \in\{, 3,5, \ldots\}$ and for some $B \in\{6,10,14, \ldots\}$ such that $u+v, u-v, C$, and $B$ coprim

$\left\{\left[u+v=p \wedge u-v=q \wedge\left(u^{2}-v^{2}\right)^{2}=(u+v)^{2}(2-v)^{2}=(p q)^{2} \Rightarrow\right.\right.$

$\left(u^{2}+v^{2}\right)^{2}-(2 u v)^{2}=\left(C^{2}\right)^{2}-\left(B^{2}\right)^{2} \wedge u^{2}+v^{2}=Q \quad \wedge 2 u v=H^{2} \wedge$

$\left(C^{2}+B^{2}=p^{2} \wedge 4 \mid B \wedge C^{2}-B^{2}=q^{2} \quad\left[2^{1}\right.\right.$ or [7] $\left.)\right]$

in view of the false proof 1 - also.

Further Weil's assumed that for some relatively $p$, ne $r, Z \in\{1,3,5, \ldots\}$ :

$\left[(2 r s \sqrt{2})^{2}=2 B^{2}=p^{2}-q^{2} \wedge=r^{2} 2 s^{2} \wedge-q=r^{2}-2 s^{2}\right.$ [7] $]$.

But this sentence cannot exi coecausc

$(2 r s \sqrt{2})^{2}=2 B^{2} \Rightarrow(s) \quad B^{2} \Rightarrow 2 \eta=B \Rightarrow 4 \nmid B$,

which is inconsiste 4 with $4 \mid B$. $\%$ is the false proof 2.

V. THE INCOR FIE PR OF OF FLT FOR $n \in \mathbb{P}$

Incomple oof. ssu $n$ e that for some $n \in \mathbb{P}$ and for some coprime

$p, q, x, x=\{1,3, \ldots\}$ such that $p>q$ and $w>r$ :

$\{[n \mid p$

$\frac{\left.(2 p q)^{n}+q^{n}\right)^{2}}{2\left(2^{n-1} q^{n}\right)}=p^{n}+2^{n-2} q^{n}=(w r)^{n}=C^{\frac{n}{2}} \wedge(w r)^{2}=C \wedge$

$\frac{(2 p q)^{n}-\left(2^{n-1} q^{n}\right)^{2}}{2\left(2^{n-1} q^{n}\right)}=p^{n}-2^{n-2} q^{n}=x^{n}=A^{\frac{n}{2}} \wedge x^{2}=A \wedge$

$\frac{(2 p q)^{n}+\left(x^{2}\right)^{n}}{2 p q+x^{2}}=\frac{(2 p q)^{n}+\left(x^{2}\right)^{n}}{\left(r^{2}\right)^{n}}=\frac{\left(w^{2} r^{2}\right)^{n}}{\left(r^{2}\right)^{n}}=\left(w^{2}\right)^{n} \wedge$

$\left.\left.\left(r^{n}\right)^{2}-x^{2}=2 p q \wedge(2 \mid p q \equiv 0)\right] \in \mathbf{0}\right\}$. 
The proof is incomplete because does not include the case for $C \in\{6,10,14, \ldots\}$ and does not include the case for $C, A \in\{3,5,7, \ldots\} \backslash\left\{3^{2}, 5^{2}, 7^{2}, \ldots\right\}$. This is the incomplete proof.

\section{THE PROOF OF THE BEAL'S CONJECTURE}

Lemma 1. For all $n \in\{3,5,7, \ldots\}$ and for all $a \in\{2,4,6, \ldots\}$ and for all $b \in\{1,3,5, \ldots\}$ and for some $c \in\{1,3,5, \ldots\}$ and for some $\mathbf{p}>2$ with $a>b$ and $\operatorname{gcd}(a, b)=1$ :

$\left[\frac{a^{n}-b^{n}}{a-b}=c \wedge \operatorname{gcd}(a,(a-b) c)=\operatorname{gcd}(b,(a-b) c)=1\right] \Rightarrow$

$(a(a-b) c)^{n}-(b(a-b) c)^{n}=((a-b) c)^{n+1}=A^{n+1}=C^{n}-B^{n} \Rightarrow$

$\mathbf{p} \mid(a-b) c, A, C, B$.

This is the lemma 1.

\section{Example 1.}

$8^{3}-7^{3}=13^{2} \Rightarrow 1352^{3}-1183^{3}=169^{4}=13^{8} \Rightarrow 104^{3}$

This is the example 1 .

Lemma 2. For all $n \in\{3,5,7, \ldots\}$ and for all $a \in\{1,5, \ldots\}$ and for all $b \in\{2,4,6, \ldots\}$ and for some $c \in\{1,3,5, \ldots\}$ and for some $\mathbf{p}>2$ with $\operatorname{gcd}(v)=1$ :

$\left[\frac{a^{n}+b^{n}}{a+b}=c \wedge \operatorname{gcd}(a,(a+b) c)=\operatorname{gcd}(b,(>\uparrow=1] \Rightarrow\right.$

$(a(a+b) c)^{n}+(b(a+b) c)^{n}=\left({ }^{2}-b\right)^{n}=C^{n+1}=A^{n}+B^{n} \Rightarrow$

p | $(a+b) c, C, A, B$.

This is the lemma 2.

Example 2.

$1^{3}+2^{3}=3\left(1+2=3 \Rightarrow 9^{3}+18^{3}=9^{4}=3^{8} \Rightarrow\left(3^{3}+6^{3}=3^{3+2}\right.\right.$ [3] $)$.

This is the examp 2 .

Lemma all $r(3,5,1, \ldots\}$ and for all $a, b \in\{1,3,5, \ldots\}$ and for some $c \in\{1,3,5, \ldots\}$ and for som $\mathbf{p}=2$ with $\mathbf{g} \mathbf{c d}(a, b)=1$ :

$\left[\frac{a^{n}+b^{n}}{a+b}=\operatorname{gcd}(a,(a+b) c)=\operatorname{gcd}(b,(a+b) c)=1\right] \Rightarrow$

$(a(a+b) c)^{n}+(b(a+b) c)^{n}=((a+b) c)^{n+1}=C^{n+1}=A^{n}+B^{n} \Rightarrow$

p | $(a+b) c, C, A, B$.

This is the lemma 3.

\section{Example 3.}

$$
1^{n}+1^{n}=2 \Rightarrow\left(2^{n}+2^{n}=2^{n+1} \quad \text { [3] }\right) \text {. }
$$

This is the example 3 . 
Lemma 4. For all $n \in\{3,5,7, \ldots\}$ and for all $a, b \in\{1,3,5, \ldots\}$ and for some $c \in\{1,3,5, \ldots\}$ and for some $\mathbf{p} \geq 2$ with $a>b$ and $\operatorname{gcd}(a, b)=1$ :

$\left[\frac{a^{n}-b^{n}}{a-b}=c \wedge \operatorname{gcd}(a,(a-b) c)=\operatorname{gcd}(b,(a-b) c)=1\right] \Rightarrow$

$(a(a-b) c)^{n}-(b(a-b) c)^{n}=((a-b) c)^{n+1}=A^{n+1}=C^{n}-B^{n} \Rightarrow$

$\mathbf{p} \mid(a-b) c, A, C, B$.

This is the lemma 4.

Lemma 5. For all $n, a \in\{3,5,7, \ldots\}$ and for all $b \in\{2,4,6, \ldots\}$ and for some $c \in$ and for some $\mathbf{p}>2$ with $a>b$ and $\operatorname{gcd}(a, b)=1$ :

$\left[\frac{a^{n}-b^{n}}{a-b}=c \wedge \operatorname{gcd}(a,(a-b) c)=\operatorname{gcd}(b,(a-b) c)=1\right] \Rightarrow$ $(a(a-b) c)^{n}-(b(a-b) c)^{n}=((a-b) c)^{n+1}=A^{n+1}=C^{n}-$ p | $(a-b) c, A, C, B$.

This is the lemma 5 .

Lemma 6. For all $n \in\{4,6,8, \ldots\}$ and for all $a, b \in\{3,5, \ldots\}$ and for some $c \in\{2,4,6, \ldots\}$ and for some $\mathbf{p} \geq 2$ with $a>b$ and $\operatorname{gcd}(a, b)=1$ :

$\left[\frac{a^{n}-b^{n}}{a-b}=c \wedge \operatorname{gcd}(a,(a-b) c)=\operatorname{gcd}(b,(\longrightarrow)=1] \Rightarrow\right.$

$(a(a-b) c)^{n}-(b(a-b) c)^{n}=((a-b) c)^{n+1}=A^{n+1}=C^{n}-B^{n} \Rightarrow$

p | $(a-b) c, A, C, B$.

This is the lemma 6.

Lemma 7. For all $n \in\{, 6$,$\} and ty all a \in\{2,4,6, \ldots\}$ and for all $b \in\{1,3,5, \ldots\}$ and for some $c \in\{1,3,5$, . nd for so $y>2$ with $a>b$ and $\operatorname{gcd}(a, b)=1$ :

$\left.\left[\frac{a^{n}-b^{n}}{a-b}=c h(a-b) c\right)=\operatorname{gcd}(b,(a-b) c)=1\right] \Rightarrow$

$(a(a-b) c)-(b(a) c)^{n}=((a-b) c)^{n+1}=A^{n+1}=C^{n}-B^{n} \Rightarrow$

p | $a-$

This is the

Corollary 1. For all $n \in\{3,4,5, \ldots\}$ and for all $a \in\{2,3,4, \ldots\}$ and for some $c \in\{1,2,3, \ldots\}$ : $\left(a\left(a^{n}-1\right)\right)^{n}-\left(a^{n}-1\right)^{n}=\left(a^{n}-1\right)^{n+1}=$ $(a c(a-1))^{n}-(c(a-1))^{n}=(c(a-1))^{n+1} \Rightarrow$ $\left[\left(a^{n}-1\right)^{2 n}+\left(a^{n}-1\right)^{2 n+1}=\left(a\left(a^{n}-1\right)^{2}\right)^{n} \quad[3]\right]$.

This is the corollary 1 . 
Lemma 8. For all $n \in\{4,6,8, \ldots\}$ and for all $a \in\{3,5,7, \ldots\}$ and for all $b \in\{2,4,6, \ldots\}$ and for some $c \in\{1,3,5, \ldots\}$ and for some $\mathbf{p}>2$ with $a>b$ and $\operatorname{gcd}(a, b)=1$ :

$\left[\frac{a^{n}-b^{n}}{a-b}=c \wedge \operatorname{gcd}(a,(a-b) c)=\operatorname{gcd}(b,(a-b) c)=1\right] \Rightarrow$

$(a(a-b) c)^{n}-(b(a-b) c)^{n}=((a-b) c)^{n+1}=A^{n+1}=C^{n}-B^{n} \Rightarrow$

$\mathbf{p} \mid(a-b) c, A, C, B$.

This is the lemma 8 .

Lemma 9. For all $n \in\{4,6,8, \ldots\}$ and for all $a \in\{1,3,5, \ldots\}$ and for all $b \in\{2,4, \ldots$, for some $c \in\{1,3,5, \ldots\}$ and for some $\mathbf{p}>2$ with $\operatorname{gcd}(a, b)=1$ : $\left[a^{n}+b^{n}=c \wedge \operatorname{gcd}(a, c)=\operatorname{gcd}(b, c)=1\right] \Rightarrow$ $(a c)^{n}+(b c)^{n}=c^{n+1}=C^{n+1}=A^{n}+B^{n} \Rightarrow \mathbf{p} \mid a^{n}+b^{n}, C, A, B$.

This is the lemma 9.

Lemma 10. For all $n \in\{4,6,8, \ldots\}$ and for all $a, b \in\{1,3,5$, an or some $\in\{1,3,5, \ldots\}$ and for some $\mathbf{p} \geq 2$ with $\operatorname{gcd}(a, b)=1$ :

$\left[a^{n}+b^{n}=2 c \wedge \operatorname{gcd}(a, c)=\operatorname{gcd}(b, c)=1\right] \Rightarrow$ $(2 a c)^{n}+(2 b c)^{n}=(2 c)^{n+1}=C^{n+1}=A^{n}+B^{n} \Rightarrow \mathbf{p}$

This is the lemma 10 .

Proof of Beal Conjecture. The above lemmas g es $1 \mathrm{lwm}$ one of the below equations $\left[\left(A^{n+1}+B^{n}=C^{n} \equiv A^{x}+B^{y} \longrightarrow\right) \vee\left(A^{n}+B^{n}=C^{n+1} \equiv A^{x}+B^{y}=C^{z}\right)\right]$, where $n, x, y, z \in\{3,4,5, \ldots$ and $C$ have the common prime factor $\mathbf{p} \geq 2$. These lemmas gives all sol ations on equation $A^{x}+B^{y}=C^{z}$ such that $A, B$, and $C$ have the common prime factor $\mathbf{p} \geq$ a the numb the solutions $[\mathrm{A}, \mathrm{B}, \mathrm{C}]$ is infinite. This is the proof.

Theorem 2 (Beal The $\mathrm{m}$ ). the strength of the proof of the Main Conjecture -

For all $x, y, z \in\{,, 5, \ldots\}$ tho atation
$A^{x}+B^{y}=C^{z}$

has no primitive sol ns in $\{1,2,3, \ldots\}$.

REE TNC $\mathrm{S}$

[1] http. wy.bealconjecture.com

[2] Dolan, s.: Fermat's method of descente infinie, Mathematical Gazette 95, July 2011, 269271

[3] http://en.wikipedia.org/wiki/Beal's_conjecture

[4] Gładki, P. : http://www.math.us.edu.pl/ ppgladki/faq/node135.html

[5] http://www.ijetae.com/files/Volume2Issue12/IJETAE_1212_14.pdf

[6] Mazur. B. : About The Cover: Diohantus's Arithmetica, http://www.ams.org/journals/bull/2006-43-03/S0273-0979-06-01123-2/S0273-0979-0601123-2.pdf

[7] Weil, A. : Fermat's Only Published Proof, http://www.cut-theknot.org/arithmetic/FermatsInfiniteDescent.shtml

[8] http://en.wikipedia.org/wiki/Fermat's_Last_Theorem 